\title{
Additional lines in quantum wells excitonic spectra connected with QW asymmetry caused by technology
}

\author{
S.M.Ryabchenko ${ }^{1}$, F.V.Kirichenko ${ }^{2}$, Yu.G.Semenov ${ }^{2}$, \\ V.G.Abramishvili ${ }^{1}$, A.V.Komarov ${ }^{1}$ \\ 1 Institute of Physics of the National of Academy Sciences of Ukraine, \\ 46 Nauki Avenue, Kiev, Ukraine \\ 2 Institute of Semiconductors Physics \\ of the National of Academy Sciences of Ukraine, \\ 45 Nauki Avenue, Kiev, Ukraine
}

Received July 7, 1998

\begin{abstract}
The calculations of intensity ratio of both the main and additional lines, the energy differences between which are fulfilled for quantum well $(\mathrm{QW})$ with asymmetrical potential profile, are presented here. It is grounded on the basis of this calculation that additional line in exciton spectrum of QW can be explained by transitions between the confined states of valence and conductivity electrons with different parity, which ceases to be forbidden in the presence of asymmetry of QW potential profile caused by technology of growth. It is shown that e1-hh2 additional exciton line is more intensive in most of the actual cases. In particular, it is shown that the additional exciton line, which was observed in the laser ablation grown structures with QW, may be explained as e1-hh2 transition. The calculations show the substantial sensitivity of the results not only to the parameter of widening of the interface, but to the detailed type of the interface profile function. It is concluded that the laser ablation method of heterostructure growth leads to a larger asymmetry of QW potential profile caused by technology than MBE potential profile.
\end{abstract}

Key words: quantum well, asymmetrical potential, interface profile function, additional exciton transitions, semimagnetic semiconductors

PACS: $78.55 . E t$

\section{Introduction}

The heterostructures with quantum wells (QW) are very interesting objects for both practical applications and for scientific investigations. Most part of scientific and applied interest to QW is connected with the energy of dimensionally quantized 
states, with penetration of wave function of electrons in these states into barrier and possibility of tunnelling of electrons between a different QW in the structure.

The magnetically mixed (or diluted magnetic, or semimagnetic) semiconductors (DMS) are unique materials with possibilities for giant spin splitting (GSS) of energy positions of the carriers by the external magnetic field. In many cases this splitting reaches the values comparable with (or larger than) a bonding energy of the states of a coupled electron (or exciton). A role of an antiferromagnetic ion-ion exchange interaction in DMS is substantial as well in the case of a high enough content of magnetic ions. In $\mathrm{A}_{1-x}^{2} \mathrm{M}_{x} \mathrm{~B}^{6}$ DMS materials ( $\mathrm{M}$ is $3 d$ group magnetic ion) it decreases a $x$-dependence of magnetization in comparison with the linear growth and for $x>0.15$ the dependence turns into falling but not increasing. These materials have also got a lot of other interesting peculiarities, which are, in the main, specified by the carrier-ion exchange interaction as well. It is the main reason for a wide spreading of preparations and studies of structures with a QW based on the DMS.

There are several different types of such structures depending on using DMS as a QW or as a barrier material. In the case of QW formed from a usual semiconductor and of barrier formed from a DMS, a GSS should take place for barrier carriers only. But the exchange induced splitting of energy positions of in-well levels of confined carriers in the external magnetic field $\mathrm{H}$ takes place too, and it arises from penetrating of the wave function of these carriers in the barrier.

The effect of "paramagnetic enhancement" of confined excitons GSS was found on such a QW as an interesting and unexpected peculiarity of the discussed QW excitonic spectra. It was established experimentally in cases of high content of magnetic ions in DMS material of the barrier that this GSS is noticeably stronger than the one calculated for corresponding rectangular QW with sharp interfaces.

Two main reasons were considered to explain the effect. The first of them is a surface effect of interface for the antiferromagnetically coupled magnetic ions in the monolayers of DMS in the barriers close to the interface. The number of magnetic neighbours in these monolayers is reduced due to the lack of magnetic ions in usual semiconducting material of QW.

The second mechanism is connected with intermixing of QW and barrier materials in the region close to the interface due to the growth process and interdiffusion.

The analysis of these mechanisms contribution to the effect value (see, for instance, $[1,2]$ ) has showed that the second of them is determinant. The numerical solution of Schroedinger equation for a one-dimensional motion of electron in arbitrary profile QW potential was used in [1,2]. A simple approach based on the perturbation theory (see for instance [3]) confirmed this conclusion as well.

The experiments with especially grown "normal" and "inverted" interfaces on the structures of $\mathrm{Cd}_{1-x} \mathrm{Mn}_{x} \mathrm{Te} / \mathrm{CdTe} / \mathrm{Cd}_{1-y} \mathrm{Mg}_{y} \mathrm{Te}$ have shown that a real profile of $x(z)$ and $y(z)$ in the regions close to the interfaces is asymmetrical due to the peculiarities of the QW growth technology.

In a such a case the parity of QW potential will be broken, and it should cause a permission of optical transitions between the confined states with different parity of wave functions of valence and conductivity electrons in QW. The observation of such 
transitions in the case of asymmetry caused by the growth technology may provide the information about the real component distribution at the interface region and control the growth technology in this way.

\section{Theory}

The optical transitions in QW are permitted in the usual case between the states with the same numbers $n$ of spatial quantization for valence and conductivity electrons respectively. But transitions between the states with different $n$ of the same parity are weakly permitted too, due to the difference of effective masses of conductivity and valence electrons as well as due to the difference of QW deepness for them. As a result, the overlap integrals of envelope of wave functions of these states are not equal to zero and it is the reason for permitting the transitions.

The loss of QW potential symmetry due to asymmetrical interface intermixing should result in nonzero values of the mentioned overlap integrals for the states with different parity as well. It will give rise to the transitions between the states of a different parity, for instance of type e1-hhn (or en-hh1) with an even $n$. In the experiments with asymmetrical half-parabolic QW on the $\mathrm{Cd}_{1-x(z)} \mathrm{M}_{n x(z)}$ Te base [4] both e1-hh2 and e2-hh1 optical transitions were observed and it enabled us to determine the value of valence band offset (VBO) for $\mathrm{Cd}_{1-x} \mathrm{Mn}_{x} \mathrm{Te} / \mathrm{CdTe}$ heterostructures as $\mathrm{VBO}=0.45$.

In many cases only the states with $n=1$ (the type of e1 and hh1 or lh1) are present in QWs of a given width and deepness for electrons of conductivity and holes ("heavy"-hh and "light"-lh respectively). But for QW, it is necessary to take into account the possibility for two types of exsitons. One of them is "usual" and is formed by electron and hole both of which are confined into QW for them. The second one is "unusual" and it is formed by one of the carriers which is confined into its QW potential and by the second carrier which is in over-well state and is confined by the Coulomb attraction of the first carrier only. Both electron and hole should be considered as a "first" and a "second" carrier and two different excitons of such a type may exist.

For the excitons of similar type the motion of the carrier in the over-well state should be considered in a joined potential of QW and an effective potential of Coulomb interaction of the carriers averaged with the wave function of the "first" carrier in a confined state and with the exciton function of relative motion of an electron and a hole. At the large distance $\left(z-z_{w 0}\right)$ from the centre of QW position $\left(z_{w 0}\right)$, the Coulomb correction is decreasing as $\left(z-z_{w 0}\right)^{-1}$ and it results in the presence of states with $n>1$ in a joined potential. The wave functions of all states in this potential should be orthogonal to one another.

The similar approach was used in [5] for interpreting the additional line in the excitonic spectrum of light reflection in $\mathrm{Cd}_{0.88} \mathrm{Mn}_{0.12} \mathrm{Te} / \mathrm{CdTe} / \mathrm{Cd}{ }_{0.88} \mathrm{Mn}_{0.12} \mathrm{Te}$. This line was arising in $\sigma^{+}$GSS spectrum only at a magnetic field $H>2 \mathrm{~T}$ and was explained by the authors of [5] as e1-hh3 additional exciton. The relative intensity of such excitons in case of symmetrical rectangular QW potential may be substantial 
for providing agreement with the experiment in the case of $H$-field induced type Itype II transition in QW potential. For the carrier spin sublevels which are connected with $\sigma^{+}$optical transition it is possible for $\mathrm{Cd}_{0.88} \mathrm{Mn}_{0.12} \mathrm{Te} / \mathrm{CdTe} / \mathrm{Cd}_{0.88} \mathrm{Mn}_{0.12} \mathrm{Te}$ structure at $H=2 \mathrm{~T}$ if $\mathrm{VBO}=0.15$. The authors of [5] treat the arising additional line in $\sigma^{+}$spectrum as the evidence of type I - type II transition in their sample and as the evidence of $\mathrm{VBO}=0.15$. This value is in contradiction with the data of $[4]$.

The analysis of intermixing effect on "paramagnetic enhancement" of GSS of e1hh1 excitons in QW $[1,2]$ showed that $\mathrm{VBO}$ value $0.3-0.4$ is more preferable than the 0.15 value. At the same time studies of $\mathrm{Cd}_{1-x} \mathrm{Mn}_{x} \mathrm{Te} / \mathrm{CdTe} / \mathrm{Cd}_{1-y} \mathrm{Mg}_{x y} \mathrm{Te} \mathrm{QW}$ with "normal" and "inverted" interfaces distinctly show that a potential profile of "rectangular" QW which was grown using the MBE method has got an asymmetry caused by the technology.

An asymmetry of QW may cause the arising of "forbidden" transitions such as e1-hh2 or e2-hh1. The overlapping integrals for such transitions should depend on the asymmetry parameters and may turn out to be large enough for observing these lines.

In many cases the deepness of QW is sufficient for the presence of hh2 or e2 states in QW even without taking into account a Coulomb transformation of hole or electron QW potential.

In the present investigation we consider the additional lines in QW exciton spectra connected with optical transitions between the states with different parity, which arise due to the asymmetry of QW potential. We will consider the case of "usual" excitons in QW only, where both of the carriers are confined by the initial QW potential without Coulomb corrections.

For the quantitative analysis of this problem we carried out the numerical solving of Schroedinger equation for a one-dimensional motion of electrons and holes (with effective masses $m_{e}^{*}$ and $m_{h h}^{*}$ respectively) in the arbitrary "sabre-like" potentials $U_{e(h h) m}(z)$. The index $m$ determines the spin (moment) projection of an electron $( \pm 1 / 2)$ or of a heavy hole $( \pm 3 / 2)$ respectively.

To describe $U_{e(h h) m}(z)$ we used the approach proposed by the authors of $[1,2]$ with determining the potential as a function of the value of Mn fractional content $x(z)$. To expose $x(z)$ for QW we used the next expression:

$$
x(z)=x_{w}+\left(x_{b}-x_{w}\right)\left\{F\left(z-z_{0}\right)+\left[1-F\left(z-z_{0}-L_{w}\right)\right]\right\},
$$

where $x_{b}$ and $x_{w}$ are the nominal contents of Mn in the barrier and QW respectively (we considered the case of $x_{w}=0$ only); $z_{0}$ and $L_{w}$ is the left interface nominal position and a nominal QW width respectively. The interface profile function $\mathrm{F}(\mathrm{z})$ is used to replace the stepped function which is used in the rectangular QW case. The $F(z)$ have a trend to 1 and 0 for the left and the right sides afar from the interface region. In the case of symmetric intermixing the $[F(z)-1 / 2]$ is an antysymmetric function and it hasn't got a definite symmetry in the other cases.

We use the different probe functions $F(z)$ for modelling the real profile of the 


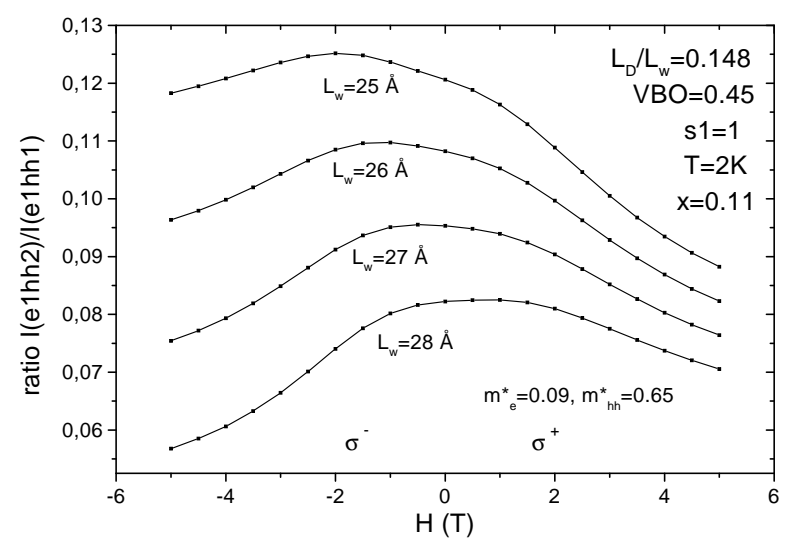

Figure 1. The H-field dependencies of $R$ of e1-hh2 and e1-hh1 transitions at different values of $L_{w}$ and fixed of $L_{D} / L_{w}$ and VBO calculated for $\sigma^{+}$and $\sigma^{-}$ GSS components ( $H>0$ and $H<0$ parts of dependencies respectively).

interface. For instance, a function of "power" type was used:

$$
F(z)= \begin{cases}1 & \text { for } z<0 \\ {\left[1+\left(z / L_{D}\right)^{s 1}\right]^{-1}} & \text { for } z \geqslant 0\end{cases}
$$

or an "exponential" function:

$$
F(z)= \begin{cases}1 & \text { for } z<0 \\ \exp \left(-z / L_{D}\right) & \text { for } z \geqslant 0\end{cases}
$$

where $L_{D}$ is a parameter of width of the intermixing region. The more complicated multi-parametrical modelling functions were tested as well. For instance, functions of a two-stage type were used which were similar to $(1,2)$, but consisted of two parts: "fast" and "slow" with weight coefficients (f) and (1-f) respectively. Each of them was characterized by its parameters such as $s 1, s 2, L_{D 1}, L_{D 2}$.

The calculations of the same type were carried out for the systems of two wells with the barrier width $L_{b}$ between them for modelling the superlattice (SL). Both electron and hole inter-well tunnelling was taken into account. The tunnel splitting of in-well states was used to evaluate the miniband width in SL with the same $L_{w}$ and $L_{b}$ as in the two-well system.

The calculation of the main (e1-hh1) and the additional (e1-hh2) exciton line energies as well as of the ratio of intensities $(R)$ of these lines was carried out for a number of possible parameters of structure materials $(x, \mathrm{VBO})$ of $\mathrm{QW}\left(L_{w}, L_{b}\right)$ and for different types of $F(t)$ or parameters of it $\left(L_{D}, s 1\right.$, etc). The $R$ was calculated as the square of a corresponding overlapping integral ratio. The dependence of it on the $H$ was considered for $\sigma^{+}$and $\sigma^{-}$components of GSS.

The calculations for a e2-hh1 optical transition were fulfilled as well. It was shown that e2-hh1 exciton line might be observable for a strong enough QW asymmetry but in all the cases examined it will be weaker than the e1-hh2 exciton line.

The results of a part of such calculations are shown on the figures 1-4 in the form of dependencies from the different QW and $F(z)$ (in the form of (2)) parameters. 


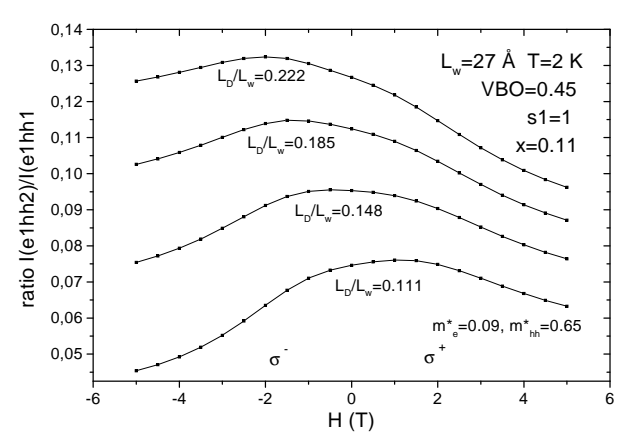

Figure 2. The calculated $H$-field dependencies of $R$ of e1-hh2 and e1hh1 transitions at different values of $L_{D} / L_{w}$ and fixed of $L_{w}$ and VBO.

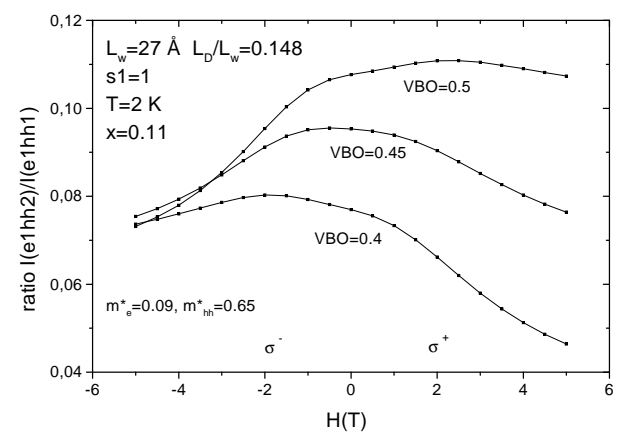

Figure 3. The calculated $H$-field dependencies of $R$ of e1-hh2 and e1-hh1 transitions at different values of $\mathrm{VBO}$ and fixed of $L_{w}$ and $L_{D} / L_{w}$.



Figure 4. The calculated $H$-field dependencies of $R$ of e1-hh2 and e1-hh1 transitions at different values of $s 1$ parameter of $F(z)$. Other parameters are fixed.

One can see that in cases of strong QW asymmetry $\left(L_{D} / L_{w}\right.$ up to 1$)$, the ratio of integral intensities of additional (e1-hh2) and main (e1-hh1) lines may be large enough (up to 10-15\%)

It is interesting to analyze the dependence of $R$ on the $H$ field for $\sigma^{+}$and $\sigma^{-}$ components of GSS. The deepness of QW decreases with $H$ for spin components of valence and conductivity electrons which are connected with $\sigma^{+}$transition and it increases for the ones connected with $\sigma^{-}$transition. From this point of view this dependence reflects the changing of the ratio with QW deepness. One can see in figures 1-4 that this dependence has got a maximum and it may take place both in $H>0$ and in $H<0$ region depending on QW and $F(z)$ parameters.

\section{Comparison with the experiment}

Light reflection spectrum measurements were fulfilled on the samples which had been grown by J.J.Dubowski (Ottawa, Canada) using the Pulsed Laser Evaporation 

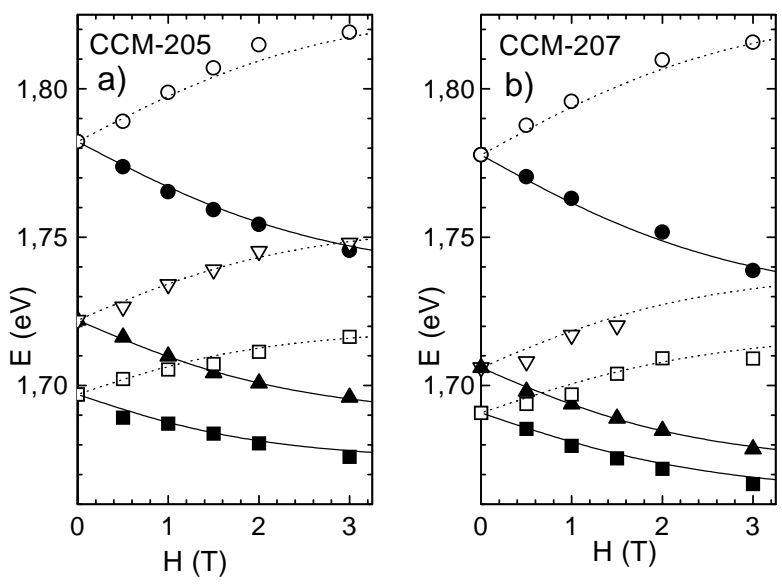

Figure 5. The $H$-field dependencies of energy of $\sigma^{+}$(full symbols) and $\sigma^{-}$(open symbols) components of GSS in CCM $205(a)$ and CCM $207(b)$ exciton reflection lines. Main line - squares, additional - triangles, barrier exciton - circles.

and Epitaxy (laser ablation) method. One of them (CCM 205) has got 6 CdTe QW with the width $L_{w}$ (the value of $L_{w}=20 \AA$ was planned in the process of the sample grown) separated by $\mathrm{Cd}_{0.89} \mathrm{Mn}_{0.11}$ Te barrier with the width $L_{b}=165 \AA$. The tunnelling of carriers across this barrier is small and the approach of a single quantum well (SQW) may be used in this case. Another structure (CCM 207) involves 41 CdTe QW with the same $L_{w}$ as in CCM 205, with the barrier of the same material, but with $L_{b}=45 \AA$. This barrier is narrow enough for inter-well tunnelling of both the conductivity electrons and the heavy holes, and the formation of a miniband results. So, this sample may be considered as a superlattice (SL). The photoluminescence measurements on these samples were carried out in [6]. The evidence of SL effects occurring was obtained. The authors of [6] suppose that the real value of $L_{w}$ in these samples is rather $27 \AA$ than $20 \AA$. Detailed data on the sample parameter are given in the mentioned reference as well.

The light reflection spectrum of these samples show the presence of additional exciton lines shifted in the high-energy side on $20 \pm 5 \mathrm{meV}$ compared to the main e1-hh1 QW exciton. This shift is stronger for CCM 205 sample than in CCM 207 sample. The GSS of the additional line is a little larger than it is for the main line and hasn't got a substantial contribution which would be proportional to $H^{2}$. It gives no possibility to connect this line to the excited states of the main exciton.

On the figures $5 a, b$ the dependencies of energies of $\sigma^{+}$and $\sigma^{-}$components of the main, the additional and the barrier excitons from $H$ at temperature $2 \mathrm{~K}$ are shown.

The additional line was distinctly observable at $H=0$ in both samples. Its intensity was up to $5-15 \%$ from the main line. The intensity of $\sigma^{+}$component of the additional line slightly increased with $H$ growth and that of $\sigma^{-}$component decreased.

One can conclude from comparing with the dependencies which are shown on figures 1-4 that these experimental data may be explained based on the mechanism 


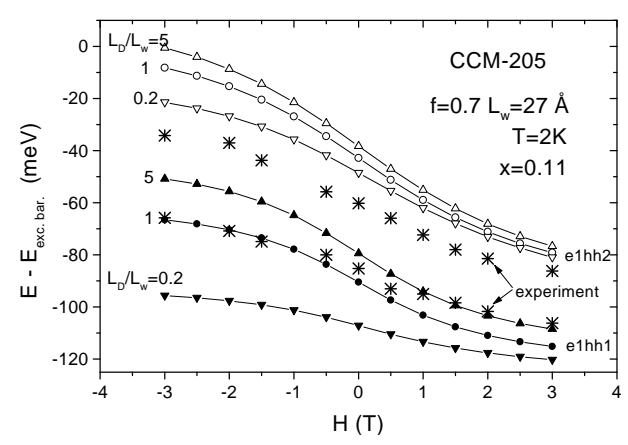

Figure 6. Comparison of CCM 205 data (stars) and calculated energy of e1-hh1 (full symbols) and e1-hh2 (open symbols) excitons versus $H$. The positions of $\sigma^{+}$and $\sigma^{-}$components corresponded to $H>0$ and $H<0$ parts of dependencies respectively. The $E_{\text {exc.bar. }}$ is the barrier exciton energy at $H=0$. The two-stage $F(z)$ (2)-like function with $s 1=2, L_{D 1} / L_{w}=0.01, s 2=1, f=$ 0.7 is used.

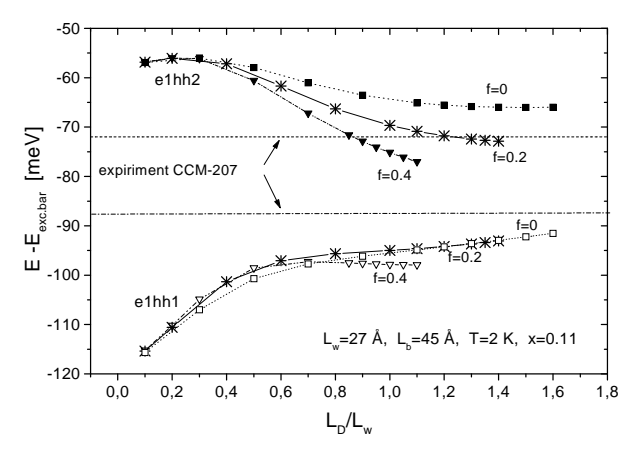

Figure 7. Comparison of CCM 207 data and energies of excitons calculated for transitions between permutative-symmetrical states into two-well potential at $H=0$ depending on $L_{D 2} / L_{w}$. The transitions, which are equivalent to e1-hh1 (full symbols) and e1-hh2 (open symbols) excitons, are taken into account. The two-stage $F(z)(2)$-like function with $s 1=2, L_{D 1} / L_{w}=0.01, s 2=1$ is used.

discussed. We should suppose that the laser ablation method leads to a strong enough asymmetry of QW profile with $L_{D} / L_{w}$ up to 1 for $L_{w} \sim 20-30 \AA$. In the MBE grown DMS based QW with close parameters the additional lines are not observable or they are weaker. It may be explained by sharper interfaces of QW grown by this method.

The calculation of the energy of the main (e1-hh1), the additional (e1-hh2) and the barrier excitons as well as of their GSS components were fulfilled in the framework of the discussed model. The best agreement with the experiment was achieved at VBO value up to 0.45 and with $F(z)$ parameters which corresponded to a strong QW asymmetry. Some results of comparing the calculations with the experiment are shown on the figures 6,7 . It's necessary to note that the calculated values of GSS, both main and additional lines, were a little larger than it was observed experimentally. It may indicate that the real intermixing of QW and the barrier material in the near interface region is a little less than it follows from the chosen $F(z)$ parameters.

The calculations carried out showed that their results are very sensitive not only to the choice of $F(z)$ parameters but to the type of this function as well. Taking into account Coulomb corrections of confining potential together with a detailed selection of $F(z)$ may be important for a more complete agreement of calculations with the experiment.

The smaller energy distance between the e1-hh1 and e1-hh2 lines in CCM 207 structure in comparison with CCM 205 is in a qualitative agreement with calculation results for a two-well model and is connected with miniband formation. 


\section{Conclusion}

The calculations of intensity ratio of both the main and additional lines are presented. Energy differences between them are fulfilled for QW with the asymmetrical potential profile. It is grounded on the basis of this calculation that additional line in exciton spectrum of QW can be explained by transitions between the confined states of valence and conductivity electrons which ceases to be forbidden in the presence of asymmetry of QW potential profile caused by technology of growth. It is shown that e1-hh2 additional exciton line is more intensive in most of the actual cases.

The calculations showed the substantial sensitivity of results not only to $L_{D}$ parameter, but to the detailed type of $F(z)$ function. It is distinct from the situation using the intermixing model for explaining a "paramagnetic enhancement" of GSS effects $[1,2]$ where only $L_{D}$ value was really important. Such a situation gives us an expectation to use the analysis of additional lines observed to obtain the information regarding the detailed interface structure at different growth technologies.

It is shown that the laser ablation method of heterostructure growth leads to a larger asymmetry of QW potential profile caused by technology than MBE potential profile.

The authors are grateful to J.J.Dubowski for providing samples of the structures for measurements as well as for a helpful discussion.

This investigation was partly supported by INTAS grant N 93-3657ext. and by the grant of State Fundamental Research Foundation of Ukraine NF4/346-97.

\section{References}

1. Gaj J.A., Grieshaber W., Bodin-Deshays C., Cilbert J., Feuillet G., Merle d'Aubigne Y., Wasiela A. Magneto-optical study of interface mixing in the CdTe- $(\mathrm{Cd}, \mathrm{Mn}) \mathrm{Te}$ system. // Phys. Rev B, 1994, vol. 50, No. 8, p. 5512-5527.

2. Grieshaber W., Haury A., Cibert J., Merle d'Aubigne Y., Wasiela A., Gaj J.A. Magnetooptical study of the interface in semimagnetic semiconductor heterostructures: Intrinsic effect and interface profile in CdTe $-\mathrm{Cd}_{1-x} \mathrm{Mn}_{x}$ Te. // Phys. Rev B, 1996, vol. 53, No. 8, p. 4891-4904.

3. Abramishvili V.G., Komarov A.V., Ryabchenko S.M., Sugakov V.I., Vertsimakha A.V. Influence of interfaces on the paramagnetic enhancement of giant spin splitting of excitonic states in structures of $\mathrm{Cd}_{1-x} \mathrm{Mn}_{x} \mathrm{Te} / \mathrm{CdTe} / \mathrm{Cd}_{1-x} \mathrm{Mn}_{x} \mathrm{Te}$. // Ukr. Fiz. Zhurn., 1998, vol. 43, No. 8, in press (in Ukrainian).

4. Kutowski M., Wojtowicz T., Grywinski G., Karczewski G., Janik E., Dynowska E., Kossut J. Half-parabolic quantum wells of diluted magnetic semiconductor $\mathrm{Cd}_{1-x} \mathrm{Mn}_{x} \mathrm{Te}$. // Acta Phys. Polonica A, 1997, vol. 92, No. 5, p. 887-890.

5. Ribayrol A., Couillat D., Lascaray J.P., Kavokin A.V., Ashenford D.E. Flare-up of the e1-hh3 exciton oscillator strength in quantum well structure under type I - type II transition. // Phys. Rev B, 1995, vol. 51, p. 7882-7890.

6. Roth A.P., Benzaquen R., Finnie P., Berger P.D., Dubowski J.J. Excitonic recombination in $\mathrm{Cd}_{0.90} \mathrm{Mn}_{0.10} \mathrm{Te} / \mathrm{CdTe}$ heterostructures grown by Pulsed Laser Evaporation and Epitaxy. // Proc. SPIE, 1994, vol. 2045, p. 322-327. 


\title{
Додаткові лінії в екситонних спектрах квантових ям,
} пов'язані з технологічно обумовленою асиметрією

\author{
С.М.Рябченко ${ }^{1}$, Ф.В.Кириченко ${ }^{2}$, Ю.Г.Семенов ${ }^{2}$, \\ В.Г.Абрамішвілі ${ }^{1}$, А.В.Комаров ${ }^{1}$ \\ 1 Інститут фізики НАН України, Київ, просп. Науки, 46 \\ 2 Інститут фізики напівпровідників НАН України, Київ, просп. Науки, 45
}

Отримано 7 липня 1998 р.

Проведені розрахунки відношення інтенсивностей основної і додаткової ліній, енергетичної відстані між ними для квантових ям (КЯ) з асиметричним потенціальним профілем. Обгрунтовано, що додаткова лінія у екситонному спектрі КЯ може бути пояснена переходами між утримуваними в КЯ станами валентних електронів і електронів провідності з різною парністю, які перестають бути забороненими у присутності асиметрії потенціального профілю, спричиненої технологією вирощування КЯ. Показано, що додаткова екситонна лінія типу е1-hh2 є більш інтенсивною в більшій частині актуальних випадків. Зокрема, показано, що додаткова екситонна лінія в структурах вирощених методом лазерної абляції, може бути пояснена як е1-hh2 перехід. Розрахунки показують суттєву чутливість не лише до параметру розширення інтерфейсу, але й до функції його профілю. Зроблено висновок, що метод лазерної абляції призводить до більшої асиметрії КЯ, ніж метод молекулярно-пучкової епітаксії.

Ключові слова: квантова яма, асиметричний потенціал, функція профілю інтерфейсу, додаткові екситонні переходи, напівмагнітні напівпровідники

PACS: $78.55 . E t$ 